\title{
Robot-Assisted Laparoscopic Surgery
}

National Cancer Institute

\section{Source}

National Cancer Institute. Robot-Assisted Laparoscopic Surgery. NCI Thesaurus. Code C116509.

A surgical technique that uses a specially designed robotic system to aid with a laparoscopic surgical procedure. 